\title{
Tracking environmental hazards and health outcomes to inform decision-making in the United States
}

\author{
Heather Strosnider, Patrick Wall, Holly Wilson, Joseph Ralph, Fuyuen Yip \\ Tracking Program, US CDC, Atlanta, Georgia, United States \\ Objective
}

To increase the availability and accessibility of standardized environmental health data for public health surveillance and decisionmaking.

\section{Introduction}

In 2002, the United States (US) Centers for Disease Control and Prevention (CDC) launched the National Environmental Public Health Tracking Program (Tracking Program) to address the challenges in environmental health surveillance described by the Pew Environmental Commission [1]. The report cited gaps in our understanding of how the environment affects our health and attributed these gaps to a dearth of surveillance data for environmental hazards, human exposures, and health effects. The Tracking Program's mission is to provide information from a nationwide network of integrated health and environmental data that drives actions to improve the health of communities. Accomplishing this mission requires a range of expertise from environmental health scienti sts to programmers to communicators employing the best practices and latest technical advances of their disciplines. Critical to this mission, the Tracking Program must identify and prioritize what data are needed, address any gaps found, and integrate the data into the network for ongoing surveillance.

\section{Methods}

The Tracking Program identifies important environmental health topics with data challenges based on the recommendations in the Pew Commission report as well as input from federal, state, territorial, tribal, and local partners. For each topic, the first step is to formulate the key surveillance question, which includes identifying the decision-maker or end user. Next, available data are evaluated to determine if the data can answer the question and, if not, what enhancements or new data are needed. Standards are developed to establish data requirements and to ensure consistency and comparability. Standardized data are then integrated into the network at national, state, and local levels. Standardized measures are calculated to translate the data into the information needed. These measures are then publically disseminated via national, state, and local web-based portals. Data are updated annually or as they are available and new data are added regularly. All data undergo a multi-step validation process that is semi-automated, routinized, and reproducible.

\section{Results}

The first set of nationally consistent data and measures (NCDM) was released in 2008 and covered 8 environmental health topics. Since then the NCDM have grown to cover 14 topics. Additional standardized data and measures are integrated into the national network resulting in 23 topics with standardized 450 measures (Figure 1). On the national network, measures can be queried via the Data Explorer, viewed in the info-by-location application, or connected to via the network's Application Program Interface (API). On average, 15,000 and 3300 queries are run every month on the Data Explorer and the API respectfully.

Additional locally relevant data are available on state and local tracking networks.

Gaps in data have been addressed through standards for new data collections, models to extend available data, new methodologies for using existing data, and expansion of the utility of non-traditional public health data. For example, the program has collaborated with the Environmental Protection Agency to develop daily estimates of fine particulate matter and ozone for every county in the conterminous US and to develop the first national database of standardized radon testing data. The program also collaborated with the National Aeronautics and Space Administration and its academic partners to transform satellite data into data products for public health.

SDS Annual Conference Proceedings 2019. This is an Open Access article distributed under the terms of the Creative Commons AttributionNoncommercial 4.0 Unported License (http://creativecommons.org/licenses/by-nc/3.0/), permitting all non-commercial use, distribution, and reproduction in any medium, provided the original work is properly cited. 
The Tracking Program has analyzed the data to address important gaps in our understanding of the relationship between negative health outcomes and environmental hazards. Data have been used in epidemiologic studies to better quantify the association between fine particulate matter, ozone, wildfire smoke, and extreme heat on emergency department visits and hospitalizations. Results are translated into measures of health burden for public dissemination and can be used to inform regulatory standards and public health interventions.

\section{Conclusions}

The scope of the Tracking Program's mission and the volume of data within the network requires the program to merge traditional public health expertise and practices with current technical and scientific advances. Data integrated into the network can be used to (1) describe temporal and spatial trends in health outcomes and potential environmental exposures, (2) identify populations most affected, (3) generate hypotheses about associations between health and environmental exposures, and (4) develop, guide, and assess the environmental public health policies and interventions aimed at reducing or eliminating health outcomes associated with environmental factors. The program continues to expand the data within the network and the applications deployed for others to access the data. Current data challenges include the need for more temporally and spatially resolved data to better unders tand the complex relationships between environmental hazards, health outcomes, and risk factors at a local level. National standards are in development for systematically generating, analyzing, and disseminating small area data and real-time data that will allow for comparisons between different datasets over geography and time.

\section{Acknowledgement}

The authors wish to acknowledge the hardwork and contributions towards the Tracking Program made by the staff, contractors, and fellows at CDC and at the state and local health departments as well as the partners in other federal programs and national organizations.

\section{References}

1. Pew Environmental Health Tracking Project Team. America's Environmental Health Gap: Why the Country Needs a Nationwide Health Tracking Network. Johns Hopkins School of Hygiene and Public Health, Department of Health Policy and Management; 2000.

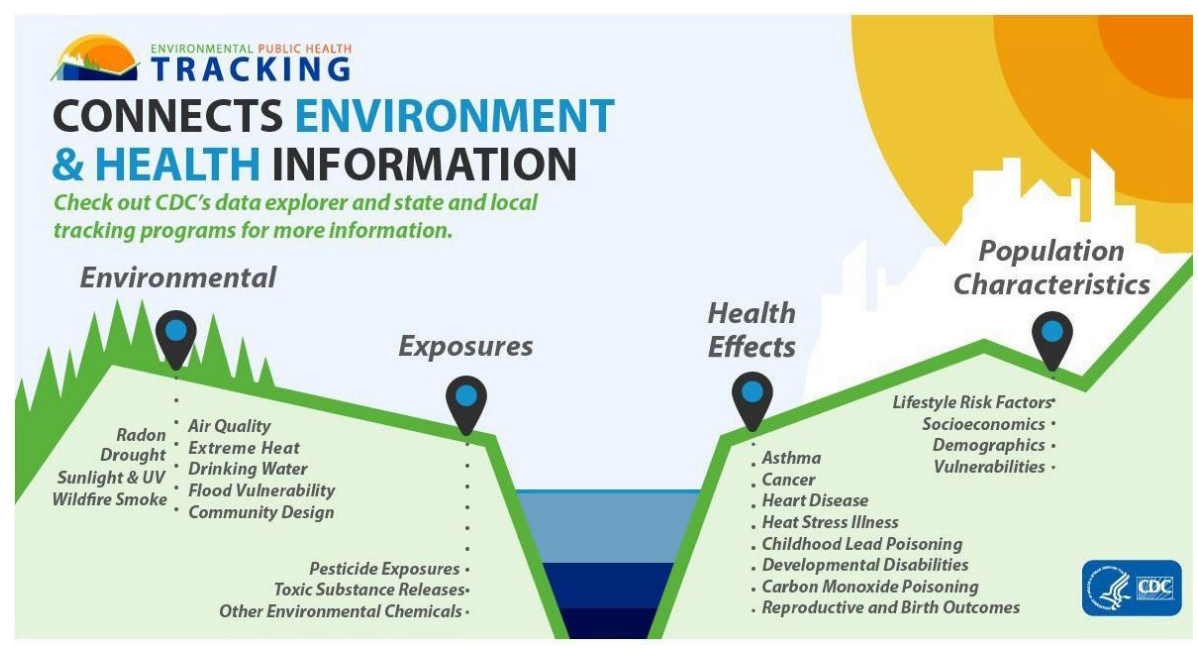

Figure 1

SDS Annual Conference Proceedings 2019. This is an Open Access article distributed under the terms of the Creative Commons AttributionNoncommercial 4.0 Unported License (http://creativecommons.org/licenses/by-nc/3.0/), permitting all non-commercial use, distribution, and reproduction in any medium, provided the original work is properly cited. 